\title{
Multidimensional DDT Modeling of Energetic Materials*
}

\author{
M. R. Baer, E. S. Hertel and R. L. Bell \\ Energetic and Multiphase Processes Dept. and Computational Shock Physics Dept. \\ Sandia National Laboratories, Albuquerque,NM, 87185
}

A nonequilibrium continuum mixture model has been incorporated into the $\mathrm{CTH}$ shock physics code to describe deflagration-to-detonation transition in granular energetic materials. This approach treats multiple thermodynamic and mechanics fields including the effects of relative material motion, rate-dependent compaction and interphase exchange of mass, momentum and energy. A finite volume description is formulated and internal state variables are solved using an operator-splitting method. Numerical simulations of low-velocity impact on a weakly-confined porous propellant bed are presented which display lateral wall release leading to curved compaction and reaction wave behavior.

\section{INTRODUCTION}

During a deflagration-to-detonation transition (DDT) event, sustaining accelerated combustion in porous energetic materials is strongly coupled to the strength of confinement. Much work has centered on experiments which have been tailored for one dimensional modeling. However, high pressure usually develops which causes significant multidimensional behavior and the effect of material motion of the confinement walls remains an unresolved issue. The study of rapid combustion in deformable confinement requires the capability of resolving multidimensional, multi-material large deformation with strong shock wave physics.

A continuum multiphase model ${ }^{1}$ has been developed to describe self-accelerated combustion in porous energetic materials. A variety of energetic materials has been experimentally and theoretically studied providing a foundation for multidimensional analyses ${ }^{2}$.

In the sections to follow, the continuum mixture formation is outlined and the numerical implementation in the shock physics code, $\mathrm{CTH}^{3}$, is described. Numerical simulations of impact on a weakly confined granular column of ball powder propellant is presented which benchmarks the mixture model. Two-dimensional simulations are presented which show the effects of lateral release at

\footnotetext{
* This work performed at Sandia National Laboratories supported by the U.S. Department of Energy under contract DE-ACO476DP00789
}

confinement walls leading to curved compaction and reaction waves.

\section{THEORETICAL DESCRIPTION}

In this study, the multiphase reactive flow model of Baer and Nunziato ${ }^{1}$ is presented; only the final forms of this model are given here. The balance equations treat a multi-component system with each phase denoted with a subscript " $a$ ". Associated with each phase are the material partial densities, $\rho_{a}=\phi_{a} \gamma_{a}$, true material density, $\gamma_{a}$, volume fractions, $\phi_{a}$, particle velocities, $\partial_{d}$, pressures, $p_{a}$, temperatures, $T_{a}$, and internal energies, $e_{a}$. The Eulerian forms of the balance laws are given as:

$$
\begin{aligned}
& \text { Mass: } \quad \grave{\mathrm{\rho}}_{a}=-\mathrm{p}_{a} \nabla \cdot \grave{\nu}_{a}+c_{a}^{\dagger} \\
& \text { Momentum: } \quad \rho_{a} \grave{\dot{D}}_{a}=\nabla \cdot \underline{\sigma}_{a}+m_{a}^{\dagger}-c_{a}^{\dagger} \dot{D}_{a}
\end{aligned}
$$

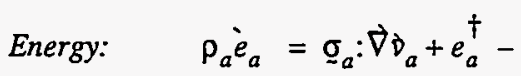

$$
\begin{aligned}
& \left(\vec{m}_{a}^{\dagger}-c_{a}^{\dagger} \vec{D}_{a}\right) \cdot \grave{D}_{a}+-c_{a}^{\dagger} E_{a}
\end{aligned}
$$

where the material derivative for each phase is de-

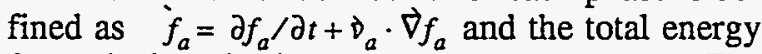
for each phase is given as $E_{a}=e_{a}+\left(\partial_{a} \cdot \partial_{a}\right) / 2$. The " $\dagger$ " superscript denotes a phase exchange quantity for mass, $c_{a}^{\dagger}$, momentum, $m_{a}^{\dagger}$, and energy, $e_{a}^{\dagger}$ and the ${ }^{a}$ stresses are represented as $\sigma_{a}=-\phi_{a} p_{a} \underline{\underline{I}}+\underline{\tau}_{a}$.

Consistent with derivations used in mixture theory, summation of each balance equation over all phases yields the response of the total mixture cor- 


\section{DISCLAIMER}

This report was prepared as an account of work sponsored by an agency of the United States Government. Neither the United States Government nor any agency thereof, nor any of their employees, make any warranty, express or implied, or assumes any legal liability or responsibility for the accuracy, completeness, or usefulness of any information, apparatus, product, or process disclosed, or represents that its use would not infringe privately owned rights. Reference herein to any specific commercial product, process, or service by trade name, trademark, manufacturer, or otherwise does not necessarily constitute or imply its endorsement, recommendation, or favoring by the United States Government or any agency thereof. The views and opinions of authors expressed herein do not necessarily state or reflect those of the United States Government or any agency thereof. 


\section{DISCLAIMER}

Portions of this document may be illegible in electronic image products. Images are produced from the best available original document. 
responding to the well known equations of motion for a single phase material. Thus, summation constraints are imposed on the ${ }_{t}$ phase interactions: $\sum c_{a}=0, \sum_{m} m_{a}=0$ and $\sum e_{a}^{\dagger}=0$. The restrictions of the Second Law of Thermodynamics also suggest admissable forms of phase interaction. For the sake of brevity, the algebraic manipulation will not be repeated here and the final forms of these interactions are given as the following:

$$
\begin{aligned}
& m_{a}^{\dagger}-c_{a}^{\dagger}\left(\grave{D}_{a}+\grave{\nu}_{i}\right) / 2+p_{i} \nabla \phi_{a}=\sum_{j} \delta_{j, a}\left(\grave{D}_{j}-\grave{\partial}_{a}\right)
\end{aligned}
$$

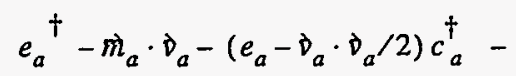

$$
\begin{aligned}
& \left(\beta_{a}-p_{a}\right)\left\{\dot{\phi}_{a}-c_{a}^{\dagger} / \gamma_{a}\right\}=\sum_{j} \mathcal{H}_{j, a}\left(T_{j}-T_{a}\right) \\
& \grave{\phi}_{a}-c_{a}^{\dagger} / \gamma_{a}=\sum_{j} \tau_{j, a}\left(p_{a}-\beta_{a}-p_{j}+\beta_{j}\right)
\end{aligned}
$$

where $\delta_{j, a}, \mathcal{H}_{j_{i} a}, \tau_{j, a}$ are exchange coefficients of positive-definite symmetric tensors reflecting interactions between phases. Interface quantities are denoted with a subscript " $i$ ". The configurational stress, $\beta_{a}$, is the stress associated with contact forces between phases and is a different stress state than the bulk stress associated with the material compressibility. Model closure is obtained by specifying an independent equation of state for each phase. Hence, the mixture description centers on the determination of appropriate constitutive relations and phase exchange coefficients using micromechanical modeling and/or experimental guidance ${ }^{1,2}$.

\section{NUMERICAL METHOD}

Having established the general equations of motion for a multiphase mixture, the conservation equations are recast into an integral form consistent with the shock physics modeling of $\mathrm{CTH}^{3}$. Overall conservation of mass, momentum and energy is preserved and relative flow effects arise as phase diffusion effects due to velocity differences between individual phase particle velocities and the mixture mass-averaged velocity, i.e. $\grave{n}_{a}=\grave{D}_{a}-\dot{D}$. Phase interaction effects, such as mass exchange, drag and heat transfer, appear as cell volume-averaged quantities. For the sake of brevity, complete derivation of the set of integral equations is not given here and details of this development, for a two-phase system, are given in Reference 4.
The phase conservation equations, given in finite volume form, have a common mathematical structure:

$$
\frac{d}{d t_{\beta}} f_{a} d V=\int_{\beta} R_{a} d V-\oint_{\partial \beta}\left(F_{a} \cdot \grave{n}\right) d S-\oint_{\partial \beta} f_{a}\left(u_{a} \cdot \grave{n}\right) d S
$$

All of the multiphase conservation equations have source and phase diffusion terms. The phase diffusion effects and cell-surface forces (the two surface integrals in Equation 7) are incorporated in an Eulerian step using operator splitting whereby all phase quantities are transported in or out of cells. The remaining cell volume source quantities, $R_{a}$, are resolved during a Lagrangian step.

During the transport step, a Flux-Corrected Transport (FCT) ${ }^{5}$ method is used to include phase diffusion effects and internal boundary conditions. This positivity-preserving high order algorithm does not introduce artificial smearing at material interfaces. It is to noted that for shocked flows, phase diffusion quantities are usually weak and important in extreme gradient regions.

Typical of multiphase simulations, the interactions of phases occur with greatly disparate timescales, leads to the solution of equations that are mathematically stiff. Since explicit time differences are inaccurate (even with subcycling), an algorithm based on asymptotic semi-analytical solutions is used for the phase interactions ${ }^{6}$. This greatly reduces computational time.

As expected, the internal state variables related to the local volume fractions must be accurately resolved to preserve consistency of the numerical solutions. Following the Lagrangian step, the volume fractions for the single mixed phase material are mapped into a single field which are then remapped back to the Eulerian mesh. These quantities are subsequently reassembled for equation of state evaluation at the beginning of the next time step. Sound speed constraints are then brought into place for evaluation of Courant conditions.

\section{IMPACT SIMULATIONS}

One-dimensional studies - A one-dimensional benchmark simulation of a piston-driven, low velocity impact on a porous bed of energetic material has been conducted which replicates conditions in experiments of Sandusky, et $\mathrm{al}^{7,8}$. A combustion gas-driven piston is impacted on a bed of nitrocellu- 
lose-nitroglycerine (NC/NG)-based ball propellant confined in a cylindrical tube geometry.

In these tests, a compaction wave develops after initial impact and high strain rate at the compaction front triggers low-level reactivity. This unstable process leads to rapid combustion and the formation of a fast deflagration wave that steepens to a shock. The interactions of compaction and multi-stage combustion are clearly complex wave processes.

One-dimensional simulations of this experiment, using the multiphase mixture model in $\mathrm{CTH}$, are displayed in Figures 1 and 2 which reproduce observations from the experimental studies. Figure 1 displays an overlay of the volume fraction of the solid phase reactant. Numerical simulation shows a dispersive compaction wave originating from the piston/bed interface that moves at a velocity consistent with experimental measurements.

As a result of high strain rate at the dispersive compaction front, low level of reactivity occurs whereby partially decomposed gas phase combustion products are formed in an induction zone. After approximately $100 \mu$ s delay, energy release in the gas phase takes place as pyrolysis gases are converted to complete product combustion gases. A secondary compaction wave then forms and is supported by combustion. When heat transfer conditions are sufficient to trigger grain burning, very rapid pressurization occurs. Eventually, the combustion wave coalesces with the primary compaction wave and an apparent abrupt change in front wave speed takes place.

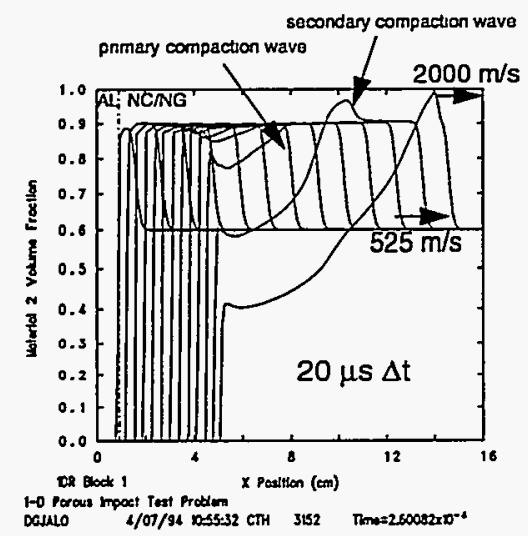

Figure 1. Overlay of solid phase volume fraction wave ficlds for a $190 \mathrm{~m} / \mathrm{s}$ impact on a porous column of ball propellant.
Details of the pressure field are shown in Figure 2. The leading jump in pressure is due to the compaction wave produced at impact and the rapid growth in pressure eventually leads to shock development prior to the coalescence of the waves. All of these features are consistent with experimental measurements.

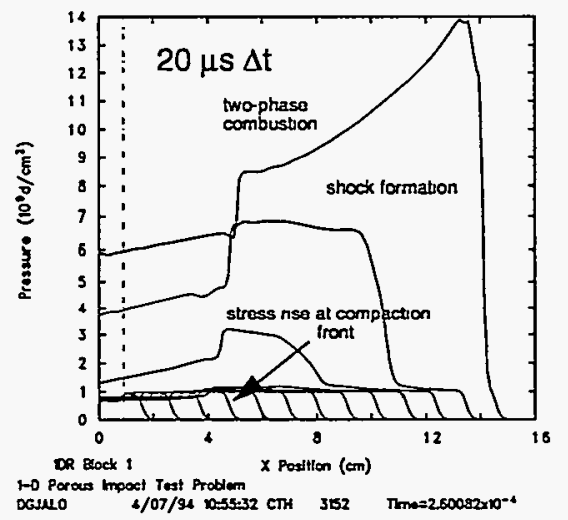

Figure 2. Overlay of pressure wave ficlds during impact and subsequent reaction in the porous propellant.

Two-dimensional simulation - In similar experimental studies conducted at Los Alamos National Laboratory by McAfee, et al. ${ }^{9}$, thin wall tubes were used in DDT tests with granular cyclotetramethylene-tetranitramine (HMX) and the release of confinement near the burning region suggested the formation of multiple "shocks" prior to the onset of rapid burning. These observations are based on sequential $X$-ray radiographs. Interpreted wave behavior was based entirely on one-dimensional jump state analysis.

A two-dimensional simulation is presented of piston impact on an energetic granular material held in weak confinement. A thin-walled steel tube (10 $\mathrm{cm}$ long, $5 \mathrm{~cm}$ outer diameter and $0.5 \mathrm{~cm}$ wall thickness), confining a NC/NG ball propellant, is modeled with impact conditions similar to the one-dimensional simulations discussed earlier. Figure 3 displays split image cross-sectional views of the confined granular energetic material at $50 \mu$ s and $150 \mu$ s after low-velocity impact. In these CTH output plots, gas pressure contours are rendered on the left half of the plots and a dot density representation of the solid phase volume fraction is shown on the right half image plane. 